\title{
Analysis of Stock Price and Volume Based on Heterogeneous Spin Model
}

\author{
Wen-Jie LIU* \\ Beijing Technology and Business University, China \\ dgliuwenjie@163.com
}

\begin{abstract}
Keywords: Stock price, Trading volume, Financial physics, Spin model, On-off intermittency, Heterogeneous investor.
\end{abstract}

\begin{abstract}
This paper introduces the heterogeneous investors based on the spin model to study the statistical characteristics and relationships between A-B stock return and trading volume during the stock-disaster period. Divide investors into fundamental traders and noise traders to match trader behaviors in the market clearing mechanism. The research results show that the volume changing is in the power-law distribution and when the magnetization volume increases, the noise trader tends to reverse the strategy, causing the trading volume to change on-off intermittency, explaining the reasons for the highly volatile trading of the bull and bear market; Although there is a positive correlation between stock returns and trading volume changes, stock returns intermittent oscillations are not significantly affected by the noise of intrinsic value fluctuations; SSE B-share investors are more sensitive to surrounding investors' attitudes than Shanghai A-share investors, and herd effects are more obvious.
\end{abstract}

\section{Introduction and Literature Review}

The rapid development of China's stock market is maturing. Investors are pouring in to seek high returns. However, mood fluctuations, irrational investment, imperfect capital structure have given certain impediments to optimizing resource allocation in the securities market. Skilled short-term investment rather than long-term value investment[1][2] took a dominant position and the importance of transaction indicators increased. The return on investment is one of the most concerned indicators for stock investors. While the change in trading volume is an important indicator of investor attitude to change the trend of returns. Many scholars have noted the necessity of studying the relationship between the two, Karpoff[3] used a simple econometric model to obtain a positive correlation between trading volume and price changes in the financial market; Imad A. Moosa[4]tested a two-way causal relationship between the volume of stocks and stock price changes in the Asian stock market; HuimingZhu[5]studied the two based on the extreme quantile regression and found that the high score points are positively correlated, the low score points are negatively correlated and the investors have herding effects; Guanjun Zhou[6]analyzed the static and dynamic relationship between volume and price based on the VaR model, and concluded that the stock price is positively correlated with the information volume and negatively correlated with the non-information volume; Lihui Tian[7] used Fama-Mac Beth regression to find that the volume risk cannot be explained by the return rate reversal effect.

There are many problems in the existing studies by sorting out: First, most of the literature is based on the analysis of the overall indicators. There is no classification and comparison. The research results are one-sided. Second, the research methods are mostly limited to financial measurement models, and the methods are relatively single; Third, heterogeneous investors, who are the main players in market participation, are lack of consideration and deeper research. In summary, this paper will study the statistical characteristics and relationships between the returns and volume of A-B shares in financial markets based on spin model and introduce heterogeneity factors in order to solve the above problems.

This paper is based on Bornholdt's spin model and adding heterogeneous investor factors, treating magnetization as a signal of trading volume, dividing investors into fundamental trader and 
noise trader, and matching the trader's behavior under the market clearing mechanism. Expect to explain the magnetization phenomenon in financial markets and study the statistical characteristics and relationships between volume and return.

\section{Data Analysis}

Select Shanghai Stock Exchange A-shares and B-shares indices and trading volume to conduct comparative analysis. In order to show the rising, falling, and smoothness of the index, it is persuasive that the time of selection is from July 2014 to November 2017 before and after the occurrence of the stock disaster. The data comes from the RESSET database. From the figure, it can be observed that the frequency of A-shares and B-shares is relatively consistent and the increase period is about $275 \%$.

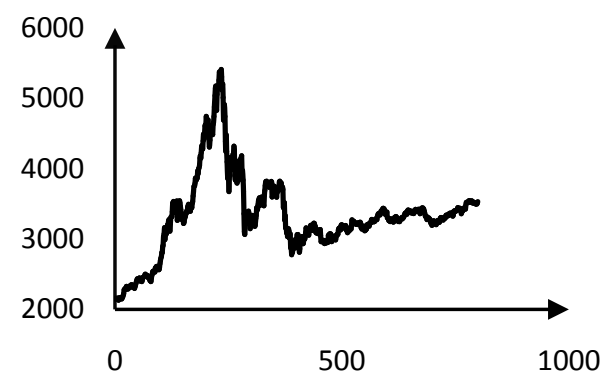

Fig.1 Shanghai Stock Exchange A-shares Index

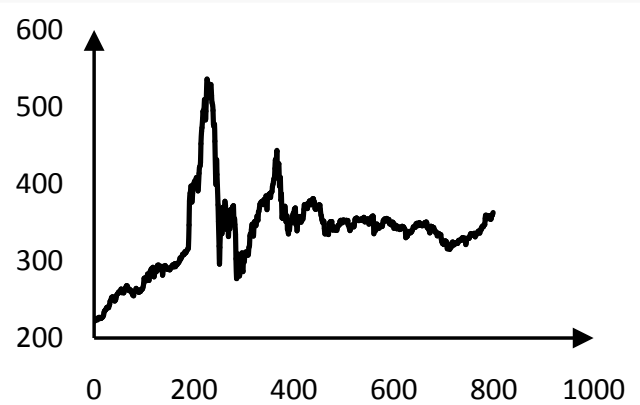

Fig.2 Shanghai Stock Exchange B-shares Index

\section{On-off Intermittency of Volume Change}

Based on the stock price index and trading volume to calculate the price logarithmic rate of return and volume changes, the formula is expressed as:

$$
\text { Eq1.2: } \quad R_{I}(t)=\ln I(t)-\ln I(t-1) C_{V}(t)=V(t)-V(t-1)
$$

Among them, $\mathrm{R}$ is the logarithmic rate of return, $\mathrm{C}$ is the change in volume, $\mathrm{I}$ and $\mathrm{V}$ represent the stock price index and volume. Take the logarithm of the stock price return rate, and take the relative amount of change in the volume, we get the time series in Figure 3-6.The trading volume changes on-off intermittency can be seen from the chart and it is more pronounced of the Shanghai B-shares. The Shanghai A-shares are affected by slight noise; while for the stock-price yield, neither of them shows significant on-off intermittency, which is greatly affected by noise.

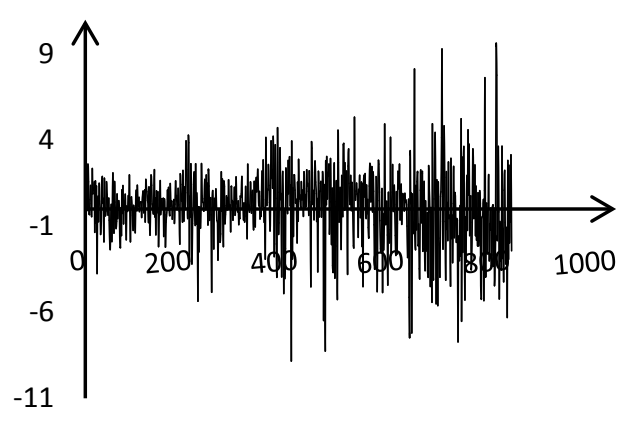

Fig. 3 SSE A-shares Index Yield

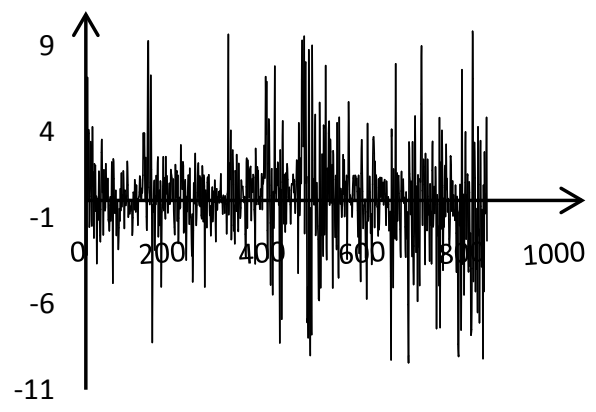

Fig. 4 SSE B-shares Index Yield 


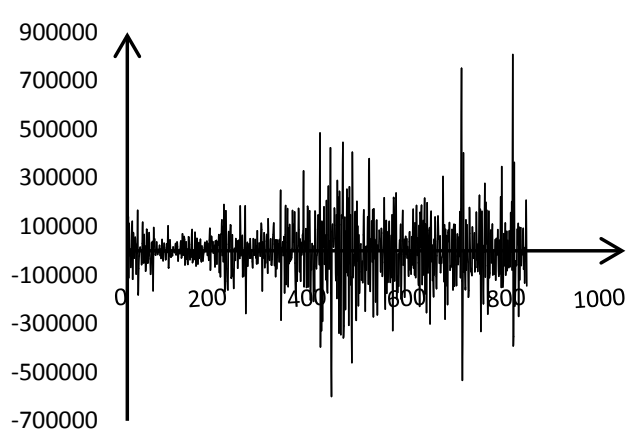

Fig. 5 Changes in volume of SSE A-shares

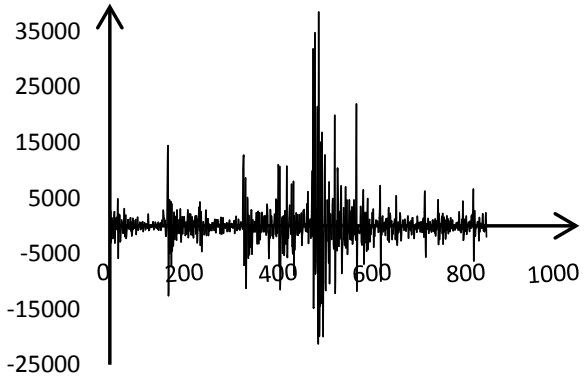

Fig. 6 Changes in volume of SSE B-shares

Compared with Shanghai A-shares and B-shares, there is still market segmentation between them. What is the difference between them? Why does the volume change show on-off intermittency? This paper starts with the relationship between volume and yields to find the answer.

\section{Correlation Analysis}

The correlation between the logarithmic returns of A-B shares and trading volume changes was analyzed and the correlation coefficients are 0.23 and 0.04.They are relevant only within one week. Absolute values of the two are found to have strong autocorrelation between the absolute changes, in which the absolute change in trading volume AC value is smaller, and the autocorrelation duration is longer, but the positive correlation is weak. The correlation coefficient between logarithmic return rate and trading volume is only 0.09 and 0.17 . Since the correlation between the two is very weak, what is the relationship between them as the two most important trading indicators in the stock market? The absolute changes in the trading volume of A-shares and B-shares both passed the power law distribution goodness-of-fit test and Zipf's law test, converged to the corresponding probability distribution of the Levy process. The 2009-2017 data was used to verify the power law distribution to ensure the universality of the results.

\section{Model Establishment and Result Analysis}

The relationship between trading volume and stock returns has been very close in the studies described in the previous article both at home and abroad. Why does the relationship between the A-shares and B-shares is weak? This article introduces Bornholdt's spin model and adds a heterogeneous investor factor to divide investors into fundamental trader and noise trader. Among them, the number of fundamental traders is $g$ and the number of noise traders is $\mathrm{k}$. Under the market clearing mechanism, the trader's behavior is matched and the magnetization is treated as a volume signal. This model is suitable for short-term financial market changeing.

\section{Fundamental Trader}

Fundamental traders focus on the long-term performance and prospects of listed companies and have a deep understanding of their intrinsic value. If the intrinsic value is lower than the market price, it is sold; when the intrinsic value is higher than the market price, it is bought. For fundamental trading groups:

$$
\operatorname{Eq5}:^{X^{F}}(t)=\operatorname{ag}\left\{\ln E\left[I^{*}(t)\right]-\ln I(t)\right\}
$$

$X^{F}$ is comprehensive performance of fundamental trading groups, $a$ is fundamental investor's sensitivity to company value. ${ }^{E\left(I^{*}\right)}$ is the intrinsic value of the company that investors expect.

\section{Noise Trader}

Assume that for a fixed period of time, the number of stocks traded by a noise trader is $b$, the trader 
notes $i$, The investment attitude of a noise trader is uncertain and is represented by the random variable $^{S}$.The investor's choice of positive (buy) attitude or negative (sale) attitude is expressed as +1 and -1 respectively in the calculation. The spin model is expressed as:

$$
\begin{array}{ll}
\text { Eq6: } & s_{i}(t+1)=+1 \text { with } p=1 /\left[1+\exp \left(-2 \beta h_{i}(t)\right)\right] \\
\text { Eq7: } & s_{i}(t+1)=-1 \text { with }(1-p)
\end{array}
$$

Formula (6)(7) gives the herding effect and noise trading in the dynamic model proposed by T. Lux (1998). Investors are affected by the attitude $h$ of the surrounding investors and the degree of influence is $\beta$, The greater of the $\beta$, the more likely it is to be influenced by the attitude of the neighbors.

Assume that the noise trader is only affected by the size of the group and the decisions made by the surrounding neighbors and the group size belongs to a minority or a majority group. The asymmetry of group fluctuations is expressed as:

Eq8:

$$
|H(t)|=\left|\frac{1}{n} \sum_{i=\alpha}^{n} s_{i}(t)\right|
$$

The noise trader chooses to enter the majority for yield, but with the increase of ${ }^{|H(t)|}$ the transaction difficulty increases obviously, and the growth rate of $\left.\right|^{|H(t)|}$ will decrease. At this point, investors in majority group are aware of the increased risk and will switch their investment direction to minority group. In other words, when the asymmetry of the population increases, the decisions of most groups will be easily reversed, while the minorities will still choose to enter the majority groups, and the minority groups will become risk takers. For the influence of the attitude of the surrounding neighbor $\mathrm{h}[21][22]$ :

$$
\text { Eq8 }
$$$$
h_{i}(t)=\sum_{j=1}^{n} J_{\forall} s_{j}(t)-\gamma s_{j}(t)|H(t)|
$$

Among them, the population scale coupling constant $\gamma>0$, the mutual influence between surrounding neighbors is represented as $\boldsymbol{J}_{i j}$ by the Ising-Hamiltonian function, and it is the interaction between the magnetic moments of the atoms. The change in volume with on-off intermittency characteristics was confirmed in equation (9). When the magnetization becomes larger, $h_{i}(t)$ tends to spin over. If the trading volume becomes larger, the noise trader tends to reverse the strategy. The noise investor's stock investment demand is:

$$
\text { Eq10: } \quad X^{I}(t)=b k H(t)
$$

\section{Stock Price and Trading Volume}

When the market clears, the stock price is determined by supply and demand, and tends to market clearance price. That is, when demand and supply are balanced:

$$
\text { Eq11: } \quad X^{F}(t)+X^{s}(t)=0=a g\left[\ln I^{*}(t)-\ln I(t)\right]+b l H(t)
$$

At this time, the stock price and volume are calculated as:

$$
\begin{array}{ll}
\text { Eq12: } & \ln I(t)=\ln I^{*}(t)+\frac{b k}{a g} H(t) \\
\text { Eq13: } & V(t)=\frac{b k(1+\mid \overline{H(t) \mid)}}{2}
\end{array}
$$

From formula (12), it can be analyzed that if $\mathrm{H}(\mathrm{t})=0$, the stock price and the intrinsic value are equal; if $\mathrm{H}(\mathrm{t})>0$, then the stock market price is higher than the company's intrinsic value, there is a bubble, manifested as bull market; $\mathrm{H}(\mathrm{t})<0$, then the stock price is lower than the intrinsic value, and the expected value is underestimated, representing bear market. When there is only fundamental 
investors in the stock market, the stock price is always equal to the intrinsic value, and the market effective hypothesis is established at this time.

$$
\text { Eq14: } \quad R_{I}(t)=\ln I(t)-\ln I(t-1)=\ln I^{*}(t)-\ln I^{*}(t-1)+\frac{b k}{a g} H(t)-\frac{b k}{a g} H(t-1)
$$

Combining (14) we can get that stock price return and trading volume change is not a purely positive or negative change relationship mentioned in previous studies, but is subjected to the fluctuation of stock intrinsic value. The effective market hypothesis proposed by Eugene F. Fama is consistent with the random walk model in price movements, which explains the discrepancy between stock price return and volume changes in Figures 3 and 6, and the reason why the correlation coefficient is weak.

Regarding the difference between A-B shares, starting from the model's premise, the noise trader's influence on the surrounding investment attitude is $\beta$, then does the A-B shares have the same sensitivity to the surrounding investment attitude? Tan[23]conducted a herd effect test on the A-B shares market investors based on the CKK model and found that the B-shares herd effect was stronger than that of the A-shares. SunJianquan[24]used the quantile regression method to reach the same conclusion, but Thomas Chiang[25] obtained the exact opposite conclusion by least squares method. In this model, $\beta$ considers the noise trader's decision based on the premise of strong sensitivity. Therefore, the change in trading volume of A shares is affected by slight noise and it can be concluded that it has little impact on the surrounding investment attitude.

\section{Conclusion and Suggestion}

This paper introduces physics into the financial market based on the spin model, takes into account investor sentiment and decision heterogeneity, and studies the A-B shares' difference of the Shanghai Stock Exchange, the statistical characteristics and relationships of the stock price return and trading volume changes. Introducing Bornholdt's spin model based on the Ising spin model is more practical, and the magnetization trading volume is treated as a signal, which is more conducive to the analysis of the relationship between volume and return. The results show that the trading volume has on-off intermittency characteristics and follows Zipf'slaw; B-shares are more sensitive to surrounding investors' decision-making, and herd effects are obvious, which are consistent with the hypothesis of this article. A-shares have weaker sensitivity, making their on-off intermittency not obvious; There is a weak positive correlation between the return of stock price and trading volume, but the intrinsic value of listed companies is an important factor affecting the change of the rate of return, leading to a random walk of stock prices, which does not meet the on-off intermittency and has a strong noise impact.

This study reveals that since the opening of B-share investments in 2001, the division of A-B shares has still existed in 16 years, and investors' information asymmetry, trading restrictions, and transaction attitudes have hindered the development of market integration; At the same time, the heterogeneity investor spin model proposed in this paper has certain significance for investors to understand the relationship between trading volume changes and return, assisting investors in decision-making.

\section{Reference}

[1] Yusong Gong. The Causes and Countermeasures of China's Stock Market Bubble [J]. Auditing and Economic Research, 2017(5):119-126.

[2] Ziyuan Zhou. Research on the Relationship Between Stock Price Volatility and Corporate Fundamental Risk in China's Listed Companies[J]. Exploration of Economic Issues, 2010(6)94-99.

[3] Karpoff J.M. The Relation between Price Changes and Trading Volume: A Survey[J].Journal of Financial and quantitative Analysis,1987(1):109-126. 
[4] ImadA. M \& Nabeel E. Al-Loughani. Testing the Price-Volume Relation in Emerging Asian Stock Markets[J]. Journal of Asian Economics,1995(3):407-422.

[5] Huiming Zhu. Research on Dependence of Stock Returns and Trading Volume Based on Extreme Quantile Regression[J].Theory and Practice in Finance, 2017(208):39-44.

[6] Guanjun Zhou. Research on Price Behavior of Chinese Stock Market Based On Volume and Price Analysis [D].Capital University of Economics and Business, 2005.

[7] Lihui Tian. Five-factor Model of China's Stock Pricing: How Does Trading Volume Affect Stock Returns? [J]. Nankai Economic Research, 2014(2): 54-75.

[8] Wanshun Qin. Empirical Study on the Difference between China A Shares and B Shares[J]. Journal of Quantitative Economics and Technology, 2000(5):15-19. 